\title{
Montaigne. Le livre III des "Essais", R. Cappellen et D. Knop (dir.)
}

\section{Filippo Fassina}

\section{Q OpenEdition}

1 Journals

\section{Edizione digitale}

URL: http://journals.openedition.org/studifrancesi/10548

DOI: 10.4000/studifrancesi. 10548

ISSN: 2421-5856

\section{Editore}

Rosenberg \& Sellier

\section{Edizione cartacea}

Data di pubblicazione: 1 décembre 2017

Paginazione: $540-542$

ISSN: 0039-2944

\section{Notizia bibliografica digitale}

Filippo Fassina, «Montaigne. Le livre III des "Essais", R. Cappellen et D. Knop (dir.)», Studi Francesi

[Online], 183 (LXI | III) | 2017, online dal 01 février 2018, consultato il 21 janvier 2021. URL: http:// journals.openedition.org/studifrancesi/10548; DOI: https://doi.org/10.4000/studifrancesi. 10548

Questo documento è stato generato automaticamente il 21 janvier 2021.

\section{(c) (i) (9)}

Studi Francesi è distribuita con Licenza Creative Commons Attribuzione - Non commerciale - Non opere derivate 4.0 Internazionale. 


\title{
Montaigne. Le livre III des "Essais", R. Cappellen et D. Knop (dir.)
}

\author{
Filippo Fassina
}

\section{NOTIZIA}

Montaigne. Le livre III des “Essais", Actes de la journée d'étude organisée à l'Université Paris Diderot le 10 décembre 2016, sur le troisième livre des Essais de Montaigne, textes réunis par Raphaël CAPPELLEN et Déborah KNOP, mis en ligne en février 2017 avec le soutien de l'Université de Lausanne, Fabula/Les colloques, Montaigne. Le livre III des Essais, URL :https://www.fabula.org/colloques/sommaire4193.php.

1 Il programma di questa giornata di studi è riassunto, nell'introduzione, con l'ausilio di due gravures dello stampatore Thomas Bertheu, che raffigurano il concetto filosofico del «conosci te stesso» attraverso l'immagine dell'ouroboros, cioè del serpente che si morde la coda. Tale concetto, frequente sia nella letteratura dell'antichità, sia in autori moderni quali Erasmo e Rabelais, è ripreso da Montaigne più volte, tanto da essere considerato una delle idee portanti della sua opera. La conoscenza di sé negli Essais, come in molte altre opere letterarie cinquecentesche, ha un ruolo fondamentale, ma ciò che rende unico Montaigne è l'humour con il quale egli tratta una questione che affonda le proprie origine nella filosofia di Platone. A partire da queste considerazioni generali, viene sviluppato un percorso analitico relativo al III libro dell'opera montaigniana, dal punto di vista linguistico e contenutistico, al fine di mettere in luce come l'autore si auto-rappresenti e come rifletta sulla propria condizione e sul ruolo che occupa nella società e all'interno della propria opera.

«D'où nous vient notre bonheur à lire Montaigne?» è l'interrogativo che si pone MarieClaire Thomine nel primo dei contributi di questi atti, Le goût de la langue: remarques sur l'usage des mots concrets dans le chapitre "De ménager sa volonté». Il rapporto tra la vita privata di Montaigne e la sua attività pubblica ha necessariamente delle implicazioni anche sullo stile utilizzato negli Essais: l'autore, applicando una sorta di détachement, 
impiega infatti uno "style comique et privé», contrapposto a quello pubblico, rivendicando così «une forme [s]ienne, inepte aux negotiations publiques». L'A. sottolinea come uno dei piaceri della lettura degli Essais stia proprio nella presenza costante dell'elemento concreto e quotidiano, inteso anche come materiale e corporale abbinato a un lessico specifico mutuato dai mestieri, dal divertimento, dai lavori più umili e dal corpo umano descritto in tutta la sua fisicità. Il goût dei lettori contemporanei è assecondato da Montaigne anche grazie a un "parler plein de suc», che ricerca un linguaggio naturale, espressivo e diretto, privo di vuota retorica, che ha come grande modello la prosa di Seneca e, in misura minore, quella di Cicerone. Soprattutto nel capitolo De ménager sa volonté, gli esempi di questo linguaggio si moltiplicano e l'A. offre un elenco delle locuzioni più significative, studiate dal punto di vista del significato e delle origini morfologiche.

3 Sempre sul linguaggio utilizzato da Montaigne nel III libro si concentra lo studio di Déborah KNOP e Romain MENINI (L'art du "provignement» dans le troisième livre des "Essais"): in particolare, viene citata Marie de Gournay che, nelle riedizioni commentate degli Essais, porta come esempio un termine specifico, il verbo provigner, mutuato dal linguaggio agricolo e impiegato anche da Ronsard, per esemplificare e simboleggiare l'audacia espressiva dei neologismi di Montaigne. Tuttavia, in un celebre passo «Sur des vers de Virgile», Montaigne dice chiaramente di essere contrario alla pratica dei neologismi. Si crea pertanto una sorta di paradosso, all'interno del quale convivono tradizionalismo e spinta verso un linguaggio personale e innovativo. Questo contributo sottolinea come, in realtà, Montaigne utilizzi in maniera massiccia i neologismi e soprattutto le parole derivate, ma in modo tale che essi vengono, per così dire, naturalizzati, proprio come succede, sottolinea l'A., per la pianta della vite, riguardo alla quale «le crochet à provigner est un outil utilisé pour maintenir couché le cep de vigne lors du provignage». La questione è quindi circoscritta a una metafora agricola che descrive l'intervento umano sulla natura nella pratica del giardinaggio e nella coltivazione. Questione cui è ricondotta in qualche modo la filosofia di Montaigne che, nel suo linguaggio, «cultive sa néologie contrôlée en glissant, par exemple, ses mots inouïs dans des structures qui les naturalisent d'autant plus». Nella seconda parte del contributo, vengono forniti numerosi esempi di questi termini novateurs che assumono un ruolo importante dal punto di vista lessicale e retorico. Nell'appendice è offerto l'elenco delle dérivations audacieuses presenti nel III libro.

4 Al capitolo 13 del III libro degli Essais è invece dedicato il testo di chantal LIAROUTZOS ( $L a$ loi et la règle dans le chapitre «De l'expérience»), capitolo che costituisce una sorta di vero $\mathrm{e}$ proprio «discours de la méthode». Il punto di partenza è la sentenza aristotelica secondo la quale «il n'est désir plus naturel que le désir de connaissance»; tuttavia la mobilité du réel sfugge alla conoscenza umana, tanto che Montaigne parla di una sorta di maladie naturelle che caratterizza l'uomo e lo porta a una ricerca incessante della conoscenza, che spesso non trova soddisfazione. Per esemplificare questa difficoltà, Montaigne si serve del linguaggio giuridico. Le leggi umane si contrappongono, per certi aspetti, alle leggi naturali, le uniche che realmente dovrebbero guidare l'agire dell'uomo, in quanto è attraverso le regole della natura che egli riesce a liberarsi delle sue tre grandi paure: quella della morte, quella della sofferenza e quella della povertà. Si crea all'interno degli Essais una sorta di contrapposizione fra lois, create dall'uomo e quindi imperfette e spesso incomprensibili, e règles, fondate sull'esperienza della natura e quindi più adatte all'esprit umano. $\mathrm{E}$ il rispetto di queste regole è anche il fondamento della comunità, in quanto «la règle est à usage interne, mais elle contribue aussi, et par 
là même, à créer la réciprocité. Elle fonde ainsi une communauté des sujets moraux». Più che le leggi, sono tali regole interiori che caratterizzano la libertà umana $\mathrm{e}$ quell'esprit général che tiene uniti gli uomini e che è anche alla base della filosofia che guida Montaigne nella scrittura dei suoi Essais.

Si incentra invece sull'uso dei pronomi dimostrativi ce, cette, ces, all'interno degli Essais di Montaigne, il lavoro di Thomas MOLLIER «J'aimerais mieux poindre que lasser»: le philosopher montanien et ses manifestations esthétiques. Variation sur un thème barthésien. L'assunto fondamentale di ogni teoria linguistica sui dimostrativi è che essi presuppongono l'esplicitazione di ciò a cui si riferiscono, ma in Montaigne spesso diventa complessa l'identificazione del referente di alcuni di questi pronomi. La corrispondenza è talmente labile che in certi casi, come l'A. sottolinea con esempi e citazioni, nelle tre principali edizioni degli Essais sono presenti note che chiariscono il contesto e il rapporto tra i termini. Ne deriva ciò che viene definito «sousdétermination chronique du texte», cioè una sorta di imprecisione ordinaria che permea il testo montaigniano, nonostante sia indubbio che esista un plan textuel che dà unità tematica all'opera, sia dal punto di vista ideologico sia da quello egologico (relativo cioè al paysage imaginaire di Montaigne). La complessità di questo rapporto tra pronomi e referenti ha reso tuttavia necessario un massiccio intervento dei commentatori, che hanno sentito il bisogno di intervenire con note al testo, finalizzate a ricostruire questo plan che spesso tende a sfuggire ai lettori. Proprio il rapporto con il lettore è un'altra questione chiave sottolineata in questo studio: Montaigne presuppone infatti due possibili reazioni al proprio testo: «un régime neutre qui provoque chez ces derniers un sentiment de lassitude» e un "régime actif selon lequel un texte plaît ou déplait mais provoque chez le lecteur un sentiment et un jugement esthétiques». In questa considerazione, l'A. riconosce una consonanza con le idee di Roland Barthes, che con il suo «J'aimerais mieux poindre que lasser», sembra proiettare sul mondo della fotografia la teoria di Montaigne sul rapporto autore/pubblico. Questo parallelismo introduce il concetto di hasard, utilizzato sia da Montaigne sia da Barthes, per definire un'arte (rispettivamente il testo e la fotografia) essenzialmente legata al momento temporale in cui è concepita, una dimension événementielle del tutto svincolata da contenuti ideologici e valida per sé stessa e nella sua immediatezza.

Marie-Luce DEMONET ( «Moi qui suis Roi de la matière que je traite»: la définition de soi dans le Livre III des Essais) analizza invece la questione dell'autoritratto all'interno dell'opera di Montaigne. In primo luogo, l'A. fa riferimento a un passo, ritenuto emblematico, del capitolo «De l'art de conférer», in cui Montaigne si definisce «Moi qui suis Roi de la matière que je traite, et qui n'en dois compte à personne», e subito dopo si descrive «debout et couché, le devant et le derrière, à droite et [à] gauche, et en tous mes naturels plis». Si tratta dunque di un esempio di riflessione su se stesso, impostata su un'immagine pittorica. Il presente saggio si concentra, in secondo luogo, sul valore della posizione iniziale, all'interno di questo passo così importante, del pronome moi, che nel Cinquecento non si trova mai sostantivato. Anche la punteggiatura, che varia a seconda delle edizioni degli Essais, è oggetto di analisi in quanto la presenza o meno della virgola che separa il pronome personale conferisce un tono diverso alla frase e pone l'accento su un termine piuttosto che su un altro. Il rapporto fra io narrante e autoritratto, descritto come se si trattasse di un dipinto vero e proprio, è l'altro punto fondamentale di questo studio. Montaigne sembra infatti porsi in una sorta di paragone con il re di Sicilia René d'Anjou (che in realtà non fu re se non in maniera teorica), e che, secondo gli studiosi, fu un pittore egli stesso e commissionò numerose opere 
d'arte. Dopo una parentesi sulla funzione e sul significato dell'autoritratto nel Cinquecento, vengono identificati i punti di contatto fra la descrizione che Montaigne dà di sé stesso come roi de la matière e le raffigurazioni dei sovrani, delle quali vengono forniti numerosi esempi, anche attraverso il supporto di link multimediali al sito della Bibliothèque Nationale de France. Soprattutto il tema della cicatrice (elemento caratterizzante del re René) è oggetto di analisi approfondita: così come quella del re «souligne sa propre humanité dérisoire devant la postérité et la divinité», allo stesso modo quella simbolica di Montaigne rappresenta le «blessures dont il tente de peindre l'histoire» e le «plis creusés par la variété de ses émotions».

7 La complessità del ritratto che Montaigne fa di se stesso è approfondita anche da Jean BALSAMO nel suo Montaigne auteur. Conscience littéraire et pratiques éditoriales dans le livre III des "Essais". L'autore offre ai lettori un'auto-rappresentazione sia dal punto di vista fisico («le portrait chauve et grisonnant d'un vieillard malade»), sia dal punto di vista sociale («un gentilhomme distingué engagé dans la vie publique»), sia dal punto di vista etico («un vir prudens»), ma non manca la caratterizzazione tipica dell'«homme au livre», inteso come lettore e come scrittore. In particolare, per chiarire il ruolo di Montaigne stesso nella redazione della sua opera, viene preso in esame il rapporto fra l'autore degli Essais e l'editore L'Angelier, relativamente al libro III, edito nel 1588. Di questo esemplare viene fornita una dettagliata storia editoriale, con particolare attenzione alla questione del privilège e delle varianti all'interno delle diverse redazioni dal 1588 al 1595. Le addizioni sono uno dei fenomeni più rilevanti, tanto che Montaigne stesso afferma: «j'ajoute, mais je ne corrige pas», ma anche le correzioni e le soppressioni caratterizzano il III libro nel raffronto tra le varie edizioni. Ciò dimostra quanto sia stato meticoloso il lavoro di rielaborazione messo in atto dall'autore, che non manca di sottolineare il fatto che eventuali imperfezioni o errori siano dovuti a negligenza altrui. Tale espediente, abbastanza comune all'epoca, ha qui la funzione di valorizzare il ruolo dell'autore, separando nettamente l'invention dalla parte tecnica e meccanica di trascrizione, affidata all'editore. L'identità dell'autore è dunque pienamente affermata da Montaigne e arricchita rispetto alla concezione tradizionale: in primo luogo «la notion d'auteur désigne pour lui en premier lieu, dans un emploi générique, ce que l'ancienne critique appelait les 'sources'». In secondo luogo, ciò che caratterizza la sua innovazione e che gli conferisce il proprio status autoriale è una sorta di autorité sociale, che dà al suo discorso una dimension publique confermata anche dall'institution éditoriale che, di fatto, permetteva a Montaigne di spingersi più in là di quanto fosse concesso ai suoi contemporanei e di «parler de soi sans pour autant prêcher dans le désert».

8 Un'analisi del III libro dal punto di vista dell'esperienza politica di Montaigne è infine condotta da Philippe DESAN (Montaigne règle ses comptes: le caractère politique du troisième livre des "Essais"). L'incarico di amministrazione della cinquième ville di Parigi fu un momento difficile nella vita di Montaigne e questo testo diventa l'occasione per tracciare un bilancio carico di amarezza e disillusione. Più in generale, la criticità del contesto politico e religioso in cui si trovava la Francia, rese ancora più complessa la posizione di Montaigne che, come sottolinea l'A., non condivise mai davvero la posizione ambigua, inaffidabile e spesso violenta di Enrico III, anche se non arrivò mai a manifestare apertamente avversità nei confronti del sovrano. L'instabilità della situazione, soprattutto dal punto di vista religioso, ebbe delle ripercussioni anche all'interno della famiglia di Montaigne, poiché alcuni dei suoi fratelli e sorelle 
abbracciarono la causa protestante. I dubbi e le incertezze della sua vita pubblica e privata si riversano dunque all'interno del III libro degli Essais, in cui si moltiplicano le considerazioni personali e i giudizi sulle sue esperienze politiche, senza che tuttavia trapeli mai del tutto l'idea che egli appartenga a una fazione piuttosto che a un'altra. Più in generale la filosofia politica di Montaigne è riassumibile nell'assunto secondo il quale è necessario «servir le roi dans la mesure du possible, sans porter préjudice à la sécurité de sa maison ni à la réputation de son nom dans sa région», visione che dimostra una concezione realistica e pratica del rapporto con il potere. Tuttavia, la sua equidistanza da tutte le posizioni in materia religiosa fa sì che Montaigne venga visto con sospetto e che si ritrovi di lì a poco di fatto isolato: questa nuova condizione implica, in primo luogo, una diminuzione dei viaggi e una volontà di ritirarsi nella vita privata e, in secondo luogo, un cambiamento anche nello stile degli Essais e una tendenza a riflettere su sé stesso e sull'importanza dell'intimità familiare. Il bilancio sulla propria esistenza diventa dunque un rimpianto della giovinezza, visto come il periodo più bello della vita e come vera e propria ammissione di non inclinazione per la vita politica e diplomatica. 